\title{
Physics-Based Closed-Loop Control of Phasing, Peak Pressure and Work Output in HCCI Engines Utilizing Variable Valve Actuation
}

\author{
Gregory M. Shaver \\ Design Division \\ Dept. of Mechanical \\ Engineering \\ Stanford University \\ Stanford, CA 94305-4021 \\ shaverestanford.edu
}

\author{
J. Christian Gerdes \\ Design Division \\ Dept. of Mechanical \\ Engineering \\ Stanford University \\ Stanford, CA 94305-4021 \\ gerdesestanford.edu
}

\author{
Matthew Roelle \\ Design Division \\ Dept. of Mechanical \\ Engineering \\ Stanford University \\ Stanford, CA 94305-4021 \\ roelledstanford.edu
}

\begin{abstract}
Homogeneous Charge Compression Ignition (HCCI) represents a promising combustion strategy for future engines. However, HCCI lacks an easily identified combustion trigger and, when achieved via variable valve actuation (VVA), includes cycle-to-cycle coupling through the exhaust gas. This makes controlling the process decidedly non-trivial. To address these issues, the development of a closed-loop controller for HCCI combustion phasing and peak pressure is outlined. Through a series of simplifications, the essential physics behind the combustion process is captured in a low-order dynamic model. This model proves amenable to a simple control structure based on dynamic feedback linearization. Results from both simulation and experiment show that cycle-to-cycle control of VVA-induced HCCI can be achieved using this physics-based approach.
\end{abstract}

\section{INTRODUCTION}

Homogeneous charge compression ignition (HCCI) holds great promise as a means to reduce $N O_{x}$ emissions in internal combustion engines. HCCI is achieved by uniformly auto-igniting a homogeneous charge through compression alone. This process leads to a low post-combustion temperature, which significantly reduces $N O_{x}$ emissions. There are several methods used to initiate HCCI, such as heating or pre-compressing the intake air ([1], [2]), trapping exhaust gases from the previous cycle by closing the exhaust valve early [3], modulating intake and exhaust flows using variable valve actuation (VVA) to re-induct exhaust from the previous cycle ([4], [3]) or some combination of these ([5], [6]).

One of the main challenges in HCCI engines is achieving the desired combustion phasing and work output, during both steady state and transient operation. HCCI has no specific event that initiates combustion, like spark in SI engines or fuel injection in diesel engines. In addition, for residual-affected HCCI, cycle-to-cycle coupling exists through the exhaust gas temperature. To address the lack of a direct combustion initiator and cycle-to-cycle dynamics, it is generally accepted that closed-loop control will be necessary.

Several approaches to closed-loop control of HCCI engines have been demonstrated ([5], [7], [1], [8]). Agrell et. al. [5] used valve timings to effectively alter the compression ratio and control phasing. Haraldsson et. al. [7] modulated the fuel amount to vary IMEP while altering the mixture ratio of two fuels to control phasing. Olsson et. al [1] took a similar approach but used compression ratio instead of fuel mixture to shift phasing. While all of these authors used tuned PID controllers, Shaver et. al. demonstrated that HCCI controllers could also be synthesized using physics-based models [8]. The technique was demonstrated for propane combustion using variable valve actuation to modulate in-cylinder pressure while holding combustion phasing constant. This paper expands upon that result to develop a physics-based approach to combined work and phasing control.

The ability to phase HCCI and control work output of the system studied is a direct consequence of being able to vary the valve timings with the VVA system. The approach in this paper is to leave the exhaust valve opening (EVO) time fixed and modulate the exhaust valve closing (EVC), intake valve opening (IVO) and intake valve closing (IVC) times to control the mass flows. IVC dictates the effective compression ratio by determining the start of compression. By modulating IVO and EVC for a given IVC, the amounts of inducted reactant and re-inducted product can be set for any given engine cycle. The three valve timings together therefore allow independent control of both the ratio of re-inducted products to fresh reactants and the effective compression ratio. By influencing the effective compression ratio, IVC determines when the reactant and re-inducted product gases begin to be compressed. This, in turn, influences combustion timing through the chemical kinetics. While the exact kinetics are quite complex, a simplified Arrhenius rate expression captures experimental data quite well ([9], [10]) and offers a very simple understanding of the process. In short, given two mixtures with identical ratios of re-inducted products and reactants at the same temperature, early IVC leads to a higher level of compression and earlier phasing of combustion. In this way, both desired phasing and load can be controlled simultaneously by varying IVO, EVC and IVC.

The first step in model-based control is the development of a mathematical system description which captures the relevant physics. A physics-based control-oriented system model for peak pressure and combustion phasing is formu- 
lated in this paper to address this need. Since the molar ratio of re-inducted products to fresh reactants can be controlled through some combination of IVO and EVC for a given IVC, this ratio is chosen as one of the inputs to the model. Additionally, since the effective compression ratio can be controlled with IVC, the IVC timing is chosen as the second control input. A mathematical relation for the peak pressure is formulated by discretizing the various processes which occur during a HCCI combustion engine cycle, then linking them together. Combustion phasing is modeled by making some simplifications to the integrated Arrhenius model presented in previous work ([9], [10]). With the control-oriented model for peak pressure and combustion phasing, feedback linearization is then used to synthesize a nonlinear controller.

In order to implement the control strategy in simulation and experiment, a map from the desired in-cylinder ratio of fresh reactant charge and re-inducted products at a given IVC valve timing to the required IVO and EVC valve timing is realized through simulation of the induction process. Implemented on a more detailed 10-state model, the control law is able to successfully control both combustion phasing and peak pressure on a cycle-to-cycle basis, therefore indirectly controlling work output. The control approach is further supported by experiments illustrating the simpler case of tracking desired peak pressure at constant phasing. The results of both simulation and experiment are very promising, demonstrating the effectiveness of a physicsbased cycle-to-cycle approach to HCCI control.

\section{Modeling ApPROACH}

The framework for modeling HCCI combustion in a simple way involves partitioning the engine cycle into five stages, as shown in Figure 1: mixing of reactant and reinducted product gases during a constant pressure, adiabatic induction process; isentropic compression to the point where combustion initiates; constant volume combustion with heat transfer to major products; isentropic expansion to the point where the exhaust valve opens and isentropic expansion through the exhaust valve. The re-inducted product temperature is directly related to the exhaust temperature from the previous cycle. The first model input, denoted $\alpha$, is the ratio of the moles of re-inducted product $N_{p}$ to the moles of inducted reactant charge $N_{r}$, so that: $\alpha \equiv N_{p} / N_{r}$. The second model input is the IVC valve timing, which dictates the volume, $V_{1}$, between the induction and compression stages. Model outputs are the peak pressure, $P_{3}$, and the volume at the constant volume combustion event, $V_{23}$, which acts as a proxy for combustion phasing. By linking the thermodynamic states of the system together, a model of peak pressure, $P_{3}$, and phasing, $V_{23}$, for residual-affected HCCI is formulated. Note that at points between stages, the cylinder volume (see Figure 1) is either known or is a model output (as is the case for $V_{23}$ ). In following development, the modeling techniques are applied to propane-fueled HCCI.
The consideration of other fuels can be made by making appropriate changes to the fuel-specific model constants.

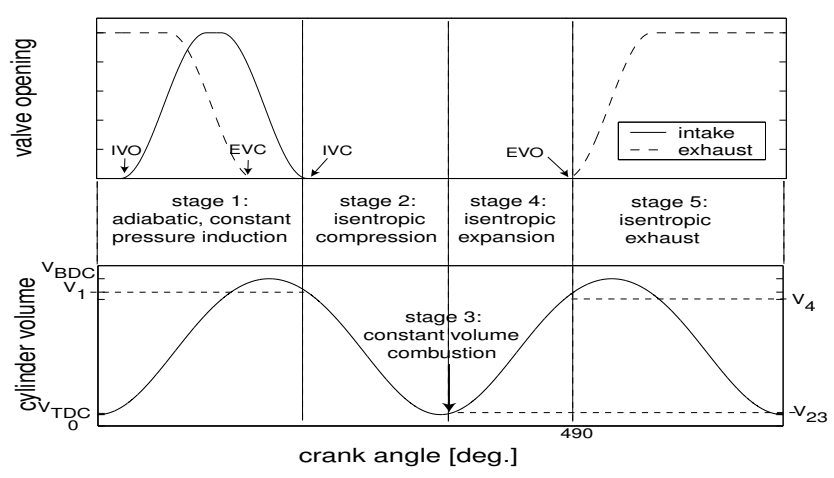

Fig. 1. General view of partitioned HCCI cycle

\section{A. Instantaneous Mixing of Species}

Using $\alpha$ as the metric for inducted gas composition, the mixing of the reactant and re-inducted product species during the induction process for lean or stoichiometric propane HCCI can be represented as:

$$
\begin{gathered}
\alpha\left(3 \phi \mathrm{CO}_{2}+4 \phi \mathrm{H}_{2} \mathrm{O}+18.8 \mathrm{~N}_{2}+5(1-\phi) \mathrm{O}_{2}\right) \\
+\left(\phi \mathrm{C}_{3} \mathrm{H}_{8}+5 \mathrm{O}_{2}+18.8 \mathrm{~N}_{2}\right) \rightarrow \\
\phi \mathrm{C}_{3} \mathrm{H}_{8}+5\left((\alpha(1-\phi)+1) \mathrm{O}_{2}+18.8(1+\alpha) N_{2}+\right. \\
3 \alpha \phi C \mathrm{O}_{2}+4 \alpha \phi \mathrm{H}_{2} \mathrm{O}
\end{gathered}
$$

where $\phi$ is the equivalence ratio, which is a measure of the relative amounts of fuel, $C_{3} H_{8}$, and oxidizer, air, in the reactant charge inducted through the intake. The first law of thermodynamics applied to an assumed adiabatic, constant pressure induction process is:

$$
\dot{m}_{\text {prod }} h_{1, p r o d}+\dot{m}_{r c t} h_{1, r c t}=\frac{d(m h)}{d t}
$$

The reactant mass flow rate through the intake and reinducted product mass flow rate through the exhaust are $\dot{m}_{r c t}$ and $\dot{m}_{\text {prod }}$, with corresponding enthalpies in the intake and exhaust manifolds of $h_{1, \text { rct }}$ and $h_{1, \text { prod }}$. When this equation is integrated from the beginning to the end of the induction process with the assumption that manifold conditions do not vary during induction, the resulting expression for the $k^{t h}$ engine cycle is:

$$
\sum_{\substack{1 \\ \text { prods. }}} N_{i, k} \bar{h}_{i}\left(T_{1 \text { prod }, k}\right)+\sum_{\substack{1 \\ \text { react. }}} N_{i, k} \bar{h}_{i}\left(T_{1 \text { rct }, k}\right)=\sum_{1} N_{i, k} \bar{h}_{i}\left(T_{1, k}\right)
$$

where $N_{i, k}$ is the number of moles of species $i, \bar{h}_{i}$ is the molar enthalpy of species $i, T_{1 \text { prod, } k}$ is the re-inducted product temperature, $T_{1 r c t, k}$ is the inducted reactant temperature and $T_{1, k}$ is the temperature of the reactants and products after full mixing. Assuming that the molar enthalpy of species $i$ can be approximated using a specific heat, $\bar{c}_{p, i}$ that is constant with temperature, then $\bar{h}_{i}(T)=\Delta_{f} \bar{h}_{i}+\bar{c}_{p, i}(T-$ $\left.T_{\text {ref }}\right)$ where $\Delta_{f} \bar{h}_{i}$ is the molar heat of formation of species $i$, and $T_{r e f}$ is the reference temperature corresponding to 
the heat of formation. Equation 3, applied to Equation 1 yields after rearrangement the following in-cylinder mixture temperature at IVC:

$$
T_{1, k}=\frac{c_{1} T_{\text {inlet }}+c_{2} \alpha_{k} T_{1 \text { prod }, k}}{c_{1}+c_{2} \alpha_{k}}
$$

where

$$
\begin{aligned}
& c_{1}=\phi \bar{c}_{p, C_{3} H_{8}}+5 \bar{c}_{p, O_{2}}+18.8 \bar{c}_{p, N_{2}} \\
& c_{2}=3 \phi \bar{c}_{p, C O_{2}}+4 \phi \bar{c}_{p, H_{2} O}+18.8 \bar{c}_{p, N_{2}}+5(1-\phi) \bar{c}_{p, O_{2}}(6)
\end{aligned}
$$

The reinducted product species are assumed to have a temperature, $T_{1 \text { prod, } k}$, that is directly related to the temperature of the exhausted products from the last cycle, $T_{5, k-1}$, as:

$$
T_{1 \text { prod }, k}=\chi T_{5, k-1}
$$

This simple relation is meant to represent heat transfer. More complex models of exhaust manifold heat transfer could be used. This expression, however, matches experimental observations reasonably well (see [8]) while keeping the relation as simple as possible. Substituting Equation 7 into Equation 4, leads to:

$$
T_{1, k}=\frac{c_{1} T_{\text {inlet }}+c_{2} \chi \alpha_{k} T_{5, k-1}}{c_{1}+c_{2} \alpha_{k}}
$$

\section{B. Isentropic Compression to Pre-Combustion State}

With the assumption that the compression stage occurs isentropically, the thermodynamic state of the system prior to and following the stage may be related with the following well known relations for an ideal gas:

$$
T_{2, k}=\left(\frac{V_{1, k}}{V_{23, k}}\right)^{\gamma-1} T_{1, k} \quad P_{2}(k)=\left(\frac{V_{1, k}}{V_{23, k}}\right)^{\gamma} P_{a t m}
$$

where $\gamma$ is the specific heat ratio.

\section{Constant Volume Combustion}

In order to model HCCI combustion in a very simple way, it is assumed that the combustion reaction phase, from reactants to products, occurs instantaneously uniformly throughout the combustion chamber. The instantaneous combustion assumption is justified by the fact that HCCI combustion is typically very fast. It is also assumed that all in-cylinder wall/piston heat transfer occurs during the combustion event. The phasing of the combustion event is modeled in Section IV. It is further assumed that only major products result from the combustion event, such that the combustion reaction can be written as:

$$
\begin{gathered}
\phi C_{3} H_{8}+5\left(\alpha_{k}(1-\phi)+1\right) O_{2}+18.8\left(1+\alpha_{k}\right) N_{2} \\
+3 \phi \alpha_{k} C O_{2}+4 \alpha_{k} \phi H_{2} O \rightarrow \\
\left(1+\alpha_{k}\right)\left(3 \phi C O_{2}+4 \phi H_{2} O+18.8 N_{2}+5(1-\phi) O_{2}\right)
\end{gathered}
$$

For a constant volume combustion process, the total internal energy before and after combustion can be related as:

$$
U_{2, k}=U_{3, k}+Q_{k}=U_{3, k}+L H V_{C_{3} H_{8}} N_{C_{3} H_{8}, k} \epsilon
$$

where the total amount of wall/piston heat transfer, $Q_{k}$, has been modeled as a certain percentage, $\epsilon$, of the chemical energy available from the combustion reaction, $L H V_{C_{3} H_{8}} N_{C_{3} H_{8}, k}$. Here $L H V_{C_{3} H_{8}}$ is referred to as the lower heating value for propane, and is defined as $L H V_{C_{3} H_{8}}=3 \Delta_{f} \bar{h}_{C_{2} O_{2}}+4 \Delta_{f} \bar{h}_{H_{2} O}-\Delta_{f} \bar{h}_{C_{3} H_{8}}$. Equation 11 can then be expanded to:

$$
\begin{gathered}
\sum_{2} N_{i, k} \bar{h}_{i}\left(T_{2, k}\right)-R_{u} T_{2, k} \sum_{2} N_{i, k}= \\
\sum_{3} N_{i, k} \bar{h}_{i}\left(T_{3, k}\right)-R_{u} T_{3, k} \sum_{3} N_{i, k}+L H V_{C_{3} H_{8}} N_{C_{3} H_{8}, k} \epsilon
\end{gathered}
$$

Applying the constant specific heat assumption to the expanded form of the post-combustion internal energy expression, Equation 12, gives:

$$
T_{3, k}=\frac{c_{3}+\left(c_{1}+c_{2} \alpha_{k}\right) T_{2, k}}{c_{2}\left(1+\alpha_{k}\right)}
$$

where:

$$
c_{3}=(1-\epsilon)\left(\phi L H V_{C_{3} H_{8}}+\left(c_{2}-c_{1}\right) T_{r e f}\right)
$$

The number of moles in the cylinder following combustion, $N_{3}$, can be related to $N_{2}$ by inspection of Equation 10 by:

$$
N_{3, k}=\left(\frac{1+\alpha_{k}}{f+\alpha_{k}}\right) N_{2, k}
$$

where $f=24.8 / 25.8$. The in-cylinder pressure following the constant volume combustion stage, $P_{3, k}$, can be related to the temperature at that point, $T_{3, k}$, by invoking the ideal gas assumption at states 2 and 3, and combining Equation 15 with Equation 9 to arrive at:

$$
P_{3, k}=\frac{1+\alpha_{k}}{f+\alpha_{k}}\left(\frac{V_{1, k}}{V_{23, k}}\right)^{\gamma} \frac{c_{1}+c_{2} \alpha_{k}}{c_{2}\left(1+\alpha_{k}\right) T_{3, k}-c_{3}} P_{a t m} T_{3, k}
$$

\section{Isentropic Expansion and Exhaust}

The fourth stage of HCCI is approximated as isentropic volumetric expansion following the constant volume combustion stage. The exhaust stage is also assumed to be isentropic, with the additional assumption that the pressure in the exhaust manifold is atmospheric. This results in the relations:

$$
\begin{gathered}
T_{4, k}=\left(\frac{V_{23, k}}{V_{4}}\right)^{\gamma-1} T_{3, k}, P_{4, k}=\left(\frac{V_{23, k}}{V_{4}}\right)^{\gamma} P_{3, k} \\
T_{5, k}=\left(\frac{P_{a t m}}{P_{4, k}}\right)^{\frac{\gamma-1}{\gamma}} T_{4, k}
\end{gathered}
$$

\section{Peak Pressure Equation}

By linking the distinct processes which occur during HCCI combustion - combining Equations 8, 9, 13, and 1618 with the approximation that $\left(1+\alpha_{k}\right) /\left(f+\alpha_{k}\right) \approx 1$ - a relation between the inputs of the system, $\alpha$ and $V_{1}$, and the peak pressure can be realized as shown in Equation 19 at the top of the next page. The presence of cycleto-cycle dynamics is evident by inspection of Equation 19. This is a very powerful expression as it relates a desired model output, the peak pressure, to the model inputs, the molar ratio of the re-inducted products and reactants, $\alpha$, and the IVC timing (via $V_{1, k}$ ). By inspection of Equation 19 it 


$$
P_{3, k}=\frac{\left(c_{3}+c_{1} T_{i}\left(\frac{V_{1, k}}{V_{23, k}}\right)^{1-\gamma}\right)\left(\frac{c_{1}}{c_{2}}+\alpha_{k-1}\right)-\left(c_{3}+c_{1} T_{i}\left(\frac{V_{1, k}}{V_{23, k}}\right)^{1-\gamma}\right)\left(\frac{V_{1, k}}{V_{23, k}}\right)^{\gamma} P_{k-1}\left(1+\alpha_{k-1}\right)-c_{3} \chi\left(\frac{V_{1, k}}{V_{23, k}}\right)^{2-\gamma}\left(\frac{V_{1, k-1}}{V_{23, k-1}}\right)^{\gamma-1} \alpha_{k} P_{k-1}^{1 / \gamma}}{\left(\frac{1+\alpha_{k}}{\frac{c_{1}}{c_{2}}+\alpha_{k}}\right)\left(c_{1} T_{i}\left(\frac{V_{1, k}}{V_{23, k}}\right)\left(\frac{c_{1}}{c_{2}}+\alpha_{k-1}\right)-c_{1} T_{i}\left(\frac{V_{1, k}}{V_{23, k}}\right)^{1+\gamma} P_{k-1}\left(1+\alpha_{k-1}\right)-c_{3} \chi\left(\frac{V_{1, k}}{V_{23, k}}\right)^{2}\left(\frac{V_{1, k-1}}{V_{23, k-1}}\right)^{\gamma-1} \alpha_{k} P_{k-1}^{1 / \gamma}\right)}
$$

can be seen that the peak pressure, $P_{3, k}$, has the following functional form:

$$
P_{3, k}=f_{1}\left(\alpha_{k}, \alpha_{k-1}, P_{3, k-1}, V_{1, k}, V_{1, k-1}, V_{23, k}, V_{23, k-1}\right)
$$

Additionally note the dependence on the combustion phasing (represented by the combustion volume, $V_{23}$ ). What is now required is a physics-based expression for the combustion phasing.

\section{Combustion Phasing Modeling Approach}

Previous work ([9], [10]) has given strong evidence that an "integrated Arrhenius model" of combustion is a simple and accurate way to mathematical describe HCCI combustion phasing. For propane fuel this integrated reaction rate, $\int R R$, takes the form:

$$
\int R R(\theta)=\int_{I V O}^{\theta} A T^{n} \exp \left(E_{a} /\left(R_{u} T\right)\right)\left[C_{3} H_{8}\right]^{a}\left[O_{2}\right]^{b} d \theta / \omega
$$

where $\omega$ is the engine speed. The values $A, E_{a} / R_{u}, a, b$ and $n$ are empirical parameters determined from combustion kinetics experiments. Once a pre-defined threshold, denoted as $K_{t h}$, for this integral is exceeded, the combustion process is initiated and assumed to proceed as a function of crank angle using a Wiebe function. The crank angle at peak incylinder pressure, $\theta_{23}$, can then be related to combustion initiation point, $\theta_{c o m b, \text { init }}$, as: $\theta_{c o m b \text {, init }}=\theta_{23}-\Delta \theta$, where $\Delta \theta$ is constant as a consequence of the combustion event proceeding as a function of crank angle. Applying the threshold approach to Equation 21, then yields:

$$
K_{t h}=\int_{I V O}^{\theta_{23}-\Delta \theta} A T^{n} \exp \left(E_{a} /(R T)\right)\left[C_{3} H_{8}\right]^{a}\left[O_{2}\right]^{b} d \theta / \omega
$$

Equation 22 captures the dependence of combustion phasing on the in-cylinder temperature, reactant concentrations and the point in which reactant charge is introduced in the cylinder (i.e IVO). Note that prior to intake valve closing (IVC) (i.e. during induction) the integrand in Equation 21 is quite small since there is no compression-induced heating or reactant concentration increase. Therefore:

$$
K_{t h} \approx \int_{\theta_{1}}^{\theta_{23}-\Delta \theta} A T^{n} \exp \left(E_{a} /(R T)\right)\left[C_{3} H_{8}\right]^{a}\left[O_{2}\right]^{b} d \theta / \omega
$$

With the aforementioned assumption of isentropic compression, the in-cylinder temperature takes the form:

$$
T_{k}=T\left(V, T_{1, k}, V_{1, k}\right)=\left(\frac{V_{1, k}}{V}\right)^{\gamma-1} T_{1, k}
$$

Before combustion and after the point in time when both the intake and exhaust valves are shut (i.e. at IVC) the reactant concentrations can be represented by inspection of Equation 1, as:

$$
\left[C_{3} H_{8}\right]_{k}=\phi N_{r, k} / V \quad\left[O_{2}\right]_{k}=5(\alpha(1-\phi)+1) N_{r, k} / V
$$

Furthermore, the total number of moles of all species is $N_{\text {total }, k}=(2 \phi+23.8) N_{p, k}+(23.8+\phi) N_{r, k}$. Then, by invoking the ideal gas assumption at IVC:

$$
N_{r, k}=\frac{P_{a t m} V_{1, k}}{R u T_{1, k}} \frac{1}{(2 \phi+23.8) \alpha_{k}+(23.8+\phi)}
$$

Substitution of Equations 8, 24-26 into Equation 23, applied to the $k^{t h}$ engine cycle, gives the equation on the top of the next page. By inspection of Equation 27 it can be seen that the volume at the combustion event, $V_{23, k}$, is a function of the molar ratio $\alpha_{k}$, the IVC timing (via $V_{1, k}$ ) and the exhaust gas temperature from the last cycle, $T_{5, k-1}$, such that:

$$
V_{23, k}=f_{2}\left(\alpha_{k}, V_{1, k}, T_{5, k-1}\right)
$$

Together, Equations 19 and 27 complete the physics-based control-oriented model of residual-affected HCCI. Although these mathematical expressions are complex nonlinear functions, they are nevertheless well-behaved and amenable for controller development.

\section{Dynamic State Feedback Linearization for CONTROL}

Through the use of the system model developed thus far a variety of closed-loop controllers can be synthesized to track desired values of peak pressure and combustion phasing. For illustrative purposes, dynamic feedback linearization [11] is used to synthesize a nonlinear tracking controller, where the desired system error dynamics are designed to be:

$$
\begin{aligned}
& e_{p, k}=\lambda_{p} e_{p, k-1},\left|\lambda_{P}\right|<1 \\
& e_{v, k}=\lambda_{v} e_{v, k-1},\left|\lambda_{V}\right|<1
\end{aligned}
$$

Here the error for the peak pressure and combustion volume at cycle $k$ are defined as $e_{p, k}=P_{3, k}-P_{3, k}^{\text {des }}$ and $e_{v, k}=$ $V_{23, k}-V_{23, k}^{\text {des }}$, respectively. These error dynamics can be re-written to show that it is desirable to achieve:

$$
\begin{array}{r}
P_{3, k}=P_{3, k}^{\text {des }}+\lambda_{P}\left(P_{3, k-1}-P_{3, k-1}^{\text {des }}\right) \\
V_{23, k}=V_{23, k}^{\text {des }}+\lambda_{V}\left(V_{23, k-1}-V_{23, k-1}^{\text {des }}\right)
\end{array}
$$


$K_{t h} \approx \frac{A(\alpha(1-\phi)+1)^{b}}{5^{-b} \phi^{-a} \omega}\left(\frac{V_{1, k}\left(c_{1}+c_{2} \alpha_{k}\right) /\left(c_{1} T_{\text {inlet }}+c_{2} \chi \alpha_{k} T_{5, k-1}\right)}{P_{a t m}^{-1} R_{u}\left((2 \phi+23.8) \alpha_{k}+(23.8+\phi)\right)}\right)^{a+b} \int_{\theta_{1, k}}^{\theta_{23, k}-\Delta \theta} \exp \left(\frac{-E_{a}\left(c_{1}+c_{2} \alpha_{k}\right)\left(V(\theta) / V_{1, k}\right)^{\gamma-1}}{R_{u}\left(c_{1} T_{\text {inlet }}+c_{2} \chi \alpha_{k} T_{5, k-1}\right)}\right) \frac{d \theta}{V(\theta)^{(a+b)}}$

The control strategy then becomes simultaneously solving

$$
\begin{array}{r}
f_{1}\left(\alpha_{k}, \alpha_{k-1}, P_{k-1}, V_{1, k}, V_{1, k-1}, V_{23, k}, V_{23, k-1}\right)= \\
P_{3, k}^{d e s}+\lambda_{P} e_{P, k-1} \\
f_{2}\left(\alpha_{k}, V_{1, k}, T_{5, k-1}\right)=V_{23, k}^{d e s}+\lambda_{V} e_{V, k-1}
\end{array}
$$

for the required system inputs: the molar ratio $\alpha_{k}$ and IVC volume $V_{1, k}$ (i.e IVC valve timing), given:

1) measurements of the previous cycles peak pressure $P_{3, k-1}$ and crank angle at peak pressure (to get $\left.V_{23, k-1}\right)$ from an in-cylinder pressure transducer.

2) an estimate or measurement of the exhaust manifold gas temperature at induction, $T_{5, k-1}$.

3) previously commanded inputs: $\alpha_{k-1}, V_{1, k-1}$

\section{VALVE TIMING MaP}

The control strategy presented determines the required intake valve closing (IVC) time and reactant/re-inducted product molar ratio $\alpha$ to track desired values of peak pressure and combustion phasing, as described in Section V. Before this can be implemented, however, a map from desired $\alpha$ for a given IVC timing to the required IVO and EVC valve timings is necessary since it is in fact the VVA system that is the control input for the engine. A map of timings can be developed directly from the compressible flow equations commonly used to model engine valves or by experiment under the assumption that exhaust temperature effects are small. The functional dependence of $\alpha$ on IVO and EVC for a given IVC then comes from a map of the form:

$$
\left.\alpha_{k}\right|_{I V C}=f_{3}(I V O, E V C, I V C)
$$

where the EVO timing is always constant at 490 CAD. A series of steady-state valve flow simulations using 1D compressible flow equations was used to generate the function $f_{3}$ is Equation 35. Inverting this relationship, the required IVO/EVC valve timings can be obtained from knowledge of the desired $\alpha$ and IVC timing. The control loop can then be closed. Figure 2 shows how the nonlinear model-based controller is used in conjunction with the valve timing map in closed loop.

\section{Controller Implementation on a 10-State MODEL OF HCCI COMBUSTION}

The controller synthesized in the previous section can be implemented on a more complete model of HCCI combustion developed previously ([9], [10]). This model is based on an open-system first-law analysis of both the in-cylinder and exhaust manifold gases, with steady state compressible flow relations used to model the mass flow through the intake and exhaust valves. Unlike the simplified

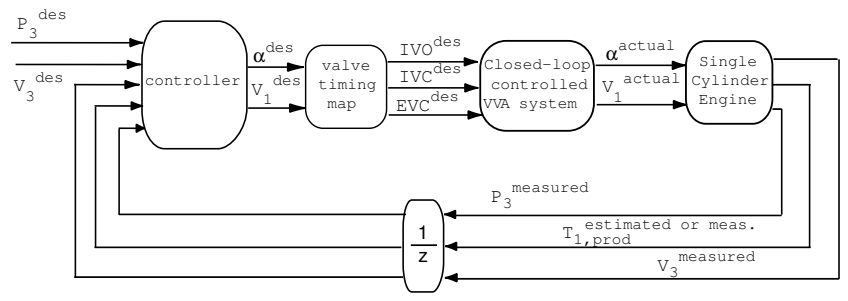

Fig. 2. Block diagram of controller implementation

model presented here, the more detailed model includes temperature dependence in the specific heats, continuous heat transfer throughout the entire engine cycle, a finiteduration combustion event captured with a Wiebe function and a control mass exhaust manifold model undergoing heat transfer.

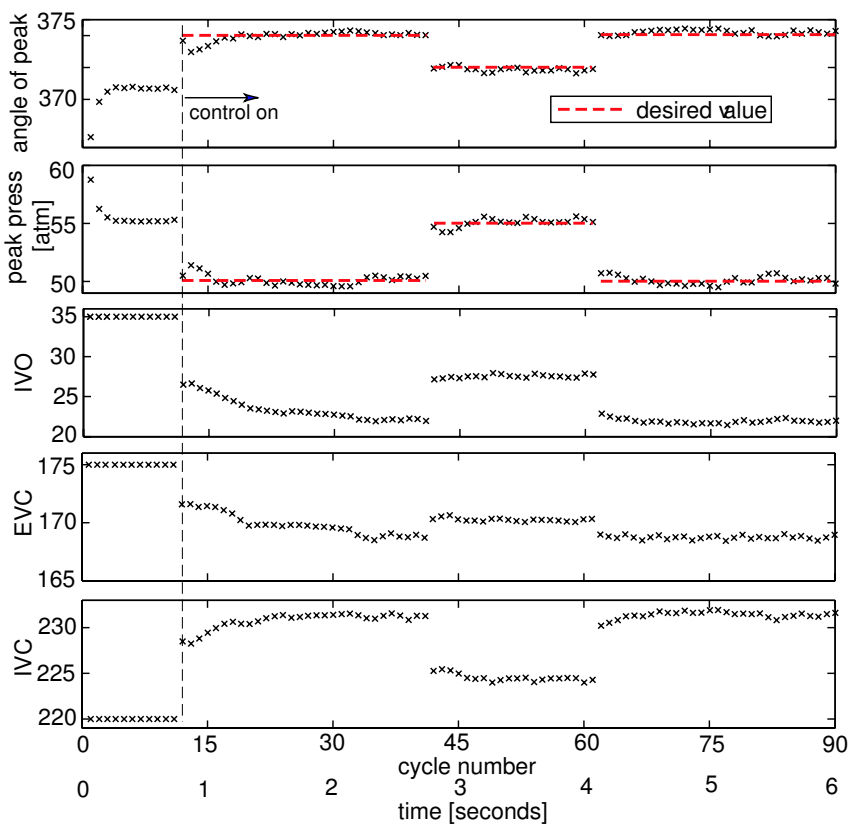

Fig. 3. Simulation of tracking controller on 10-state model, dashed line: desired values

Figure 3 shows the result of the simulation as the desired peak pressure and combustion phasing go through a series of step changes. Note that the system response to step changes is immediate, exhibiting system control on a cycle-to-cycle basis. This is a result of the physics-based approach used for controller synthesis. Since the controloriented system model has been formulated to represent the cycle-to-cycle dynamics in residual-affected HCCI, a carefully synthesized controller can be expected to exhibit control on a cycle-by-cycle basis. Additionally, note that the fluctuations in the valve timings are very manageable, 
further validating the control approach. The existence of a small amount of fluctuation in the peak pressure and angle of peak about the desired values is simply the result of simplifications in the control-oriented model.

\section{EXPERIMENTAL RESULTS}

Physics-based closed-loop control has also been implemented on a single cylinder research engine (for engine details see [4]). For these preliminary experiments, IVC is held constant, resulting in a fairly constant combustion phasing as long as the IVO/EVC timings lie along a particular linear map (see [8] and [4] for details on this map). Peak pressure control was realized by synthesizing a Linear Quadratic Regulator (LQR) controller from a linearization of Equation 19 [8]. Results for this approach are shown in Figure 4 and demonstrate both mean tracking and a substantial reduction in the cyclic dispersion compared to the uncontrolled case. The controller introduces more stability, and as a result, expands the applicable operating range for HCCI. These results verify that physics-based models can be used to synthesize controllers which operate on a cycle-to-cycle basis.

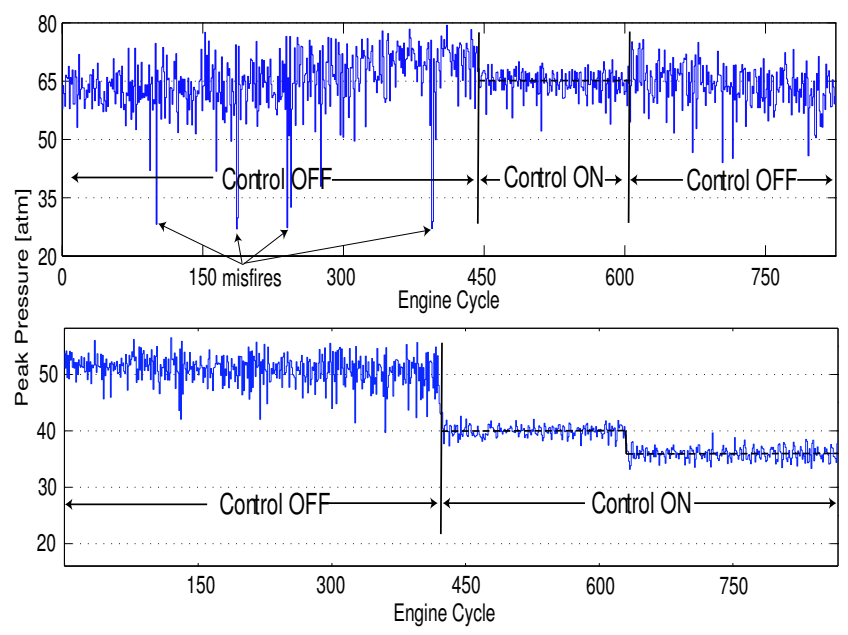

Fig. 4. Experimental control results, dashed lined - desired peak pressure, top: response to no change in desired mean pressure, bottom: response to step changes in desired peak pressure

\section{CONCLUSIONS}

While the cycle-to-cycle dynamics and chemical kinetics of VVA-induced HCCI are complex, the essential characteristics for control can be captured in a relatively low-order model. The model leads naturally to a feedback linearizing controller that uses the molar ratio of reactants to products and the the intake valve closing time as inputs. Paired with a map from desired values of the molar ratio and IVC to required IVO/EVC timing, this represents a complete approach for implementing control.
A closed-loop simulation with a more complex 10state HCCI model shows that despite a number of simplifications, the control strategy is quite effective. When implemented on an engine, a simpler strategy based on this modeling approach works quite well without additional tuning of control gains. The results from both simulation and experiment show that cycle-to-cycle control of VVAinduced HCCI can be achieved using a physics-based approach.

\section{Future WORK}

The next step in this work is the experimental implementation of the control strategy presented in Section $\mathrm{V}$ for tracking both peak pressure and combustion phasing independently. Following that, the focus will turn to relaxing some of the restrictions of the current approach, including constant engine speed and single cylinder operation.

\section{ACKNOWLEDGMENTS}

The authors would like to thank the Robert Bosch Corporation Research and Technology Center for their financial and technical support of this work. In particular, ongoing technical discussions with Dr. Jean-Pierre Hathout, Dr. Jasim Ahmed and Dr. Aleksandar Kojic have been highly appreciated and helpful.

\section{REFERENCES}

[1] J.-O. Olsson, P. Tunestal, and B. Johansson, "Closed-loop control of an HCCI engine," SAE 2001-01-1031, 2001.

[2] J. Martinez-Frias, S. M. Aceves, D. Flowers, J. R. Smith, and R. Dibble, "HCCI engine control by thermal management," $S A E$ 2000-01-2869, 2000.

[3] D. Law, D. Kemp, J. Allen, G. Kirkpatrick, and T. Copland, "Controlled combustion in an IC-engine with a fully variable valve train," SAE 2001-01-0251, 2001.

[4] P. Caton, A. Simon, J. Gerdes, and C. Edwards, "Residual-effected homogeneous charge compression ignition at low compression ratio using exhaust reinduction," Intl. J. of Eng. Res., vol. 4, no. 2, 2003.

[5] F. Agrell, H.-E. Angstrom, B. Eriksson, J. Wikander, and J. Linderyd, "Transient control of HCCI through combined intake and exhaust valve actuation," SAE 2003-01-3172, 2003.

[6] J. Hyvönen, G. Haraldsson, and B. Johansson, "Supercharging HCCI to extend the operating range in a multi-cylinder VCR-HCCI engine," SAE 2003-01-3214, 2003.

[7] G. Haraldsson, P. Tunestal, B. Johansson, and J. Hyvonen, "HCCI combustion phasing with closed-loop combustion control using variable compression ratio in a multi cylinder engine," JSAE 20030126, 2003.

[8] G. M. Shaver and J. C. Gerdes, "Cycle-to-cycle control of HCCI engines," ASME Intl. Mech. Eng. Cong. and Expo., IMECE200341966, 2003.

[9] G. M. Shaver, M. Roelle, and J. C. Gerdes, "Multi-cycle modeling of HCCI engines," To Appear in: First IFAC Sym. on Adv. in Auto. Control, Paper no: 95, 2004.

[10] G. M. Shaver, M. Roelle, J. C. Gerdes, P. A. Caton, and C. F. Edwards, "Multi-cycle modeling of HCCI engines utilizing variable valve actuation," To Appear in: ASME J. of Dyn. Sys., Meas., and Control, 2003.

[11] H. G. Lee, A. Arapostathis, and S. I. Marcus, "Linearization of discrete-time systems via restricted dynamic feedback," IEEE Trans. on Auto. Control, vol. 48, no. 9, pp. 1646-1650, 2003. 